\title{
Calidad de vida de familias de niños y adolescentes con discapacidad asociada a defectos congénitos*
}

\section{Quality of Life of Family of Children and Teenagers with Disability Associated to Birth Defects}

Recepción: 10 Septiembre 2015 | Aprobación: 20 Junio 2017

\author{
Diana Marcela Ortiz-Quiroga ${ }^{\mathrm{a}}$ \\ Universidad Icesi, Colombia \\ ORCID: http://orcid.org/0000-0003-0165-9109 \\ Yoseth Ariza \\ Universidad Icesi, Colombia \\ Harry Pachajoa \\ Universidad Icesi, Colombia
}

a Autor de correspondencia. Correo electrónico: dianaortiz9@hotmail.com

Para citar este artículo: Ortiz-Quiroga, M., Ariza, Y., \& Pachajoa, H. (2018). Calidad de vida de familias de niños y adolescentes con discapacidad asociada a defectos congénitos. Universitas Psychologica, 17(1), 1-10. https://doi.org/10.11144/Javeriana.upsy17-1.c vfn

\section{RESUMEN}

Este trabajo pretendió determinar el estado de la Calidad de Vida Familiar de niños y adolescentes con discapacidad asociada a anomalías congénitas, asistentes a la consulta de genética clínica de un centro de referencia del suroccidente colombiano. Se empleó el modelo de Calidad de Vida Familiar propuesto por la Universidad de Kansas, y la Escala de Calidad de Vida Familiar (ECVF) adaptada para Colombia. El instrumento evaluó los Indicadores y Factores de la escala. Cada factor fue evaluado en términos de importancia y satisfacción asignados por el cuidador. Se configuró el Mapa de Calidad de vida Familiar (MCVF), que señaló dos áreas (de necesidades y fortalezas). La información fue almacenada y procesada con el programa Excel. El MCVF mostró que gran parte de los indicadores se ubicaron en el área fuerte del mapa, a excepción de algunos relacionados con los Recursos familiares y Apoyo a la persona con discapacidad (PCD), que se ubicaron en el área de necesidades. Por medio de estrategias de diseño, se generó una forma gráfica para sintetizar los resultados y presentarlos de forma clara a las familias.

Palabras clave

calidad de Vida Familiar; Anomalías congénitos; modelo biopsicosocial. 
with disabilities (PWD), which were located in the needs area. Through design strategies it was generated a graphic form to summarize the results and present them clearly to families.

Keywords

family Quality of Life; birth defects; biopsicosocial model.

Las intervenciones llevadas a cabo con personas con discapacidad han evolucionado a través de la historia como consecuencia de la transición de un modelo médico-rehabilitador, centrado en la deficiencia, a un modelo bio-psicosocial, que asume la discapacidad como un acontecimiento en el mundo cotidiano, que involucra otros sistemas sociales y comunitarios, con una influencia bidireccional. (Vanegas \& Gil, 2007).

Para este modelo, la familia se constituye en el principal sistema abierto de interacción con otros contextos, y una red de apoyo determinante para el desarrollo de cada uno de los miembros que la componen (Vanegas \& Gil, 2007), con una influencia en el bienestar individual y colectivo.

En consecuencia, diferentes estudios, a nivel internacional (Summers, Hoffman, Marquis, Turnbull, \& Poston, 2005; Resch et al., 2010) y nacional (Córdoba, Verdugo, \& Gómez, 2006; Córdoba, Mora, Bedoya, \& Verdugo, 2007; Córdoba, Gómez, \& Verdugo, 2008; Aya \& Córboda, 2013), han centrado su interés en la calidad de vida de todos los miembros de la familia en el marco del Modelo de Calidad de Vida Familiar (Park et al., 2003; Park, Turnbull, \& Turnbull, 2002).

Este modelo atribuye un papel importante a la familia para la satisfacción de sus necesidades, señalando que "una familia experimenta calidad de vida en la medida en que sus miembros logran cubrir sus necesidades, disfrutan de su vida juntos y cuentan con oportunidades para seguir y alcanzar metas que son trascendentales para ellos" (Park et al., 2002), apartándose de la visión reducida de la atención sobre las necesidades individuales del miembro con discapacidad.

Sin embargo, las investigaciones sobre Calidad de Vida Familiar desde una perspectiva holística y multidimensional, se han centrado principalmente en población con discapacidad intelectual (Fernández, Montero, Martínez, Orcasitas, \& Villaescusa, 2015), y los estudios en población con anomalías congénitas se han centrado en perspectivas perceptuales de la calidad de vida o del bienestar psicológico (Ballesteros, Novoa, Muñoz, Suarez, \& Zarante, 2006), que reducen la complejidad de la situación, dado que esta podría variar según factores personales y socio- culturales (Schalock $\&$ Verdugo, 2002, 2003).

Las Anomalías congénitas son malformaciones morfológicas que pueden ser identificadas antes, durante o después del nacimiento (EUROCAT, 2012), y se consideran, desde 1994, como la segunda causa de muerte en menores de un año en el Valle del Cauca y Colombia (Fernández \& Zarante, 2007).

Por lo tanto, indagar en la calidad de vida de las familias con un miembro con diagnóstico de anomalías congénitas, desde la perspectiva del modelo de calidad de vida familiar, permitirá ir más allá del reconocimiento del impacto negativo de la condición de salud sobre las dinámicas familiares, a ubicar en otro lugar a la familia; un lugar activo en el que se reconozcan sus fortalezas y potencialidades para afrontar situaciones que aparecen cotidianamente en la relación que se tiene con los otros contextos de interacción.

Dada la importancia que tiene la comprensión de la calidad de vida desde la perspectiva de las propias familias, y la incipiente investigación en el contexto colombiano, esta investigación se propone describir la satisfacción con la calidad de vida de familias que conviven con una persona con discapacidad (niños y adolescentes), asociada a una anomalía congénita, en el suroccidente colombiano.

\section{Material y método}

Diseño

Estudio transversal. 


\section{Participantes}

Se incluyeron 40 familias de niños y adolescentes, pacientes de la consulta de genética clínica de la Fundación Valle del Lili, una institución que ofrece servicios de atención de alta complejidad, y que funciona como centro de referencia para el sur occidente colombiano. Particularmente, la Fundación cuenta con una consulta de genética clínica que ofrece atención especializada a la población pediátrica con diversas condiciones genéticas. La selección de los participantes fue por conveniencia. Se obtuvo una muestra no probabilística en la que la selección dependió de causas relacionadas con las características de la investigación, en este caso la conveniente accesibilidad y proximidad de los sujetos para el grupo investigador. Los incluidos residen en Cali o sus alrededores. En el periodo de captación, se invitó a participar de forma voluntaria a 55 sujetos que asistieron a la consulta de genética clínica -consulta que se realiza una vez por semana, y en la que se atienden 7 pacientes-, de los cuales aceptaron 40, lo que corresponde a una proporción de respuesta del 72\%. La invitación a participar se realizó en un periodo de 3 meses a cuidadores de niños y adolescentes entre 0-18 años de edad, de ambos sexos, sin restricción de raza, lugar de residencia, estrato socioeconómico o tipo de defecto congénito. Los pacientes tuvieron un diagnóstico de anomalía congénita comprobado bioquímica o molecularmente, y de todos se obtuvo un consentimiento informado.

\section{Instrumento}

Se utilizó la escala de Calidad de Vida Familiar (ECVF) adaptada para Colombia (Verdugo, Córdoba, \& Gómez, 2006), en la cual se combinan la importancia y la satisfacción con la intención de establecer la calidad de vida familiar como la diferencia entre las expectativas y los logros de la familia, de tal forma que el bienestar se alcanza cuando están satisfechas las necesidades que son importantes para esta.

Esta escala está constituida en la misma forma y estructura dimensional de su versión original en inglés, construida por el equipo del Beach Center on Disability de la Universidad de Kansas (Poston et al., 2003). Presenta 41 ítems que permiten determinar la satisfacción con la calidad de vida de las familias a través de 5 factores: Interacción Familiar, Rol Parental, Salud y Seguridad, Recursos Generales, Apoyo a las personas con discapacidad, que se califican en términos de satisfacción desde muy satisfecho (5) a muy insatisfecho (1), y en términos de importancia, desde muy importante (5) a muy poco importante (1). El primer factor, con nueve criterios de evaluación de calidad de vida, y los cuatro factores restantes, con ocho criterios cada uno.

El Factor 1, Interacción familiar, se refiere a la relación que tienen los miembros de la familia entre sí, y el clima emocional dentro del cual existe esta relación. El Factor 2, Rol parental, evalúa la forma en que los adultos proveen orientación o guía, disciplina y enseñanza a los niños y adolescentes de la familia. El factor 3, Salud y seguridad, corresponde a la salud física y mental de la familia, y las condiciones de los contextos físicos dentro de los cuales vive la misma. En el Factor 4, Recursos Familiares, los indicadores de este factor miden aspectos como el cuidado familiar, actividades diarias del hogar, obtención de ayuda externa, habilidades y oportunidades para tener relaciones con personas de fuera de la familia, contar con ingresos que le permitan a la familia por lo menos cubrir los gastos, identidad, respeto, reducción del estrés, y libre elección. El Factor 5, Apoyo para la persona con discapacidad, corresponde a las oportunidades de inclusión social con las que cuenta la persona con discapacidad, para participar en diferentes actividades como educación, trabajo y tiempo libre, así como en las actividades que llevan a cabo los miembros de la familia para apoyarse mutuamente u obtener apoyo de otros.

Con la información arrojada por la ECVF, se configuró el Mapa de Calidad de Vida Familiar (MCVF), adaptado a Colombia por Verdugo et al. (2006), que es una herramienta complementaria a la escala, y que constituye un insumo para la intervención a familias. El MCVF muestra gráficamente las intersecciones entre 
satisfacción (filas) e importancia (columnas). Una vez localizados los indicadores en el mapa, se identifican aquellos ubicados en el área crítica (muy importante, importante y muy insatisfecho, insatisfecho) o fuerte (muy importante, importante y muy satisfecho, satisfecho). Estas dos categorías corresponden a la clasificación actual de necesidades/prioridades y fortalezas de las familias, respectivamente.

\section{Procedimiento}

El protocolo de la investigación fue aprobado por el comité de ética de la Fundación Clínica Valle del Lili (IRB-EC 00652) y la universidad Icesi, los cuales consideraron esta investigación "sin riesgo" para los participantes según la declaración de Helsinki y la resolución 008430 del Ministerio de Salud de Colombia. En un primer momento, las familias fueron contactadas a través de la consulta de genética, y su participación fue autorizada a través de la firma de un consentimiento informado con el que se explicó el objetivo del estudio y las implicaciones de su participación.

En un segundo momento, se aplicaron los instrumentos de recolección de información, después de la consulta de genética clínica en sesiones que tenían una duración de 90 a 120 minutos. En un tercer momento, se ingresaron los datos a una hoja de cálculo en Excel y se generaron tablas de frecuencias absolutas y relativas.

En un cuarto momento, una vez completado el MCVF, se procedió a formular recomendaciones específicas para cada uno de los criterios y, por medio de estrategias de diseño, se generó una forma gráfica para sintetizar los resultados individuales y presentarlos de forma clara a las familias. Finalmente, el proceso de entrega de resultados se llevó a cabo a través de visitas domiciliarias a las 40 familias. Para estas recomendaciones se definió un compromiso y plan de seguimiento en un plazo de tres meses.

\section{Resultados}

\section{Características sociodemográficas}

Se aplicó la Escala de Calidad de Vida Familiar a un grupo de 40 familias atendidas en la consulta de genética de la Fundación Clínica Valle del Lili de la ciudad de Santiago de Cali. Se realizó una distribución por rangos de edad de las personas con discapacidad, ubicándose 8 niños en el grupo de 0-1 año de edad, 11 en el grupo de 2-6 años, 11 en el de 7-12 años, y 10 adolescentes en el grupo de 13-18 años. La distribución de los pacientes por género fue $52 \%$ mujeres y $48 \%$ hombres.

La Tabla 1 resume las variables sociodemográficas de la muestra. Se hallaron diferencias significativas entre las personas incluidas escolarmente que presentan una edad menor a 6 años, y aquellos mayores de 7 años. Adicionalmente, el 25\% de los pacientes en edad escolar no asiste a ninguna institución educativa.

\section{Tabla 1}

Variables socio-demográficas de las personas con discapacidad

\begin{tabular}{|c|c|c|c|c|}
\hline & \multicolumn{4}{|c|}{ Grupos de edad } \\
\hline & $\begin{array}{c}0-1 \text { Año } \\
\mathrm{n}=8\end{array}$ & $\begin{array}{c}2-6 \text { años } \\
n=11\end{array}$ & $\begin{array}{c}7-12 \text { años } \\
\mathrm{n}=11\end{array}$ & $\begin{array}{c}13-18 \text { años } \\
n=10\end{array}$ \\
\hline \multicolumn{5}{|l|}{ Ciudad de residencia } \\
\hline Área urbana & 62.5 & 82 & 64 & 80 \\
\hline Área rural & 38 & 18 & 36 & 20 \\
\hline \multicolumn{5}{|l|}{ Tipo de malformación } \\
\hline Enfermedades Neuro-genéticas & 12 & 36 & 27 & 40 \\
\hline $\begin{array}{l}\text { Síndromes genéticos asociados a } \\
\text { talla baja }\end{array}$ & 0 & 9 & 9 & 0 \\
\hline $\begin{array}{l}\text { Síndromes genéticos asociados a } \\
\text { malformaciones cráneo-faciales }\end{array}$ & 38 & 18 & 27 & 20 \\
\hline Defectos del tubo neural DTN & 25 & 18 & 36 & 40 \\
\hline Síndrome de Down & 25 & 0 & 0 & 0 \\
\hline $\begin{array}{l}\text { Síndrome genético asociado a } \\
\text { cardiopatía congénita }\end{array}$ & 0 & 18 & 0 & 0 \\
\hline \multicolumn{5}{|l|}{ Género } \\
\hline Femenino & 50 & 64 & 45 & 50 \\
\hline Masculino & 50 & 36 & 55 & 50 \\
\hline \multicolumn{5}{|l|}{$\begin{array}{l}\text { Tipo de institución educativa a la } \\
\text { que asisten }\end{array}$} \\
\hline Regular & NA & 18 & 64 & 50 \\
\hline Institución de educación especial & NA & 27 & 9 & 40 \\
\hline Ambas & NA & 0 & 9 & 10 \\
\hline Sin escolarizar & NA & 55 & 27 & 10 \\
\hline
\end{tabular}

Adicionalmente, se obtuvo que el principal cuidador o respondiente de la entrevista de Calidad de Vida Familiar fue la madre (63\%), seguido por la participación de ambos padres (28\%), solo el padre (8\%), otro familiar (3\%). El 4 $8 \%$ de los respondientes han cursado estudios superiores, y el (43\%) alcanzó la educación básica secundaria. Respecto a la situación laboral, 
el $(63 \%)$ se encuentra inactivo, mientras que el (25\%) trabaja tiempo completo, y el (12\%) se clasifica como desempleados. La ocupación prevalente fue la relacionada con las labores del hogar en un $(63 \%)$.

Por otro lado, se observa que las familias entrevistadas se encuentran conformadas predominantemente por tres $(38 \%)$ o cuatro personas (40\%), incluyendo a los niños y/o jóvenes con anomalías congénitas. El estado civil predominante de los padres o cuidadores se ubicó en la categoría de casados, con un (45\%), seguido por el de unión libre con un (30\%), y el 18\% se encuentra divorciado. Los ingresos económicos mensuales en el hogar que se obtienen para cubrir los gastos de toda la familia se ubican mayoritariamente en $3 \mathrm{SMLV}^{1}$, con un (55\%), seguido por el (35\%) con 1 SMLV.

Nivel de satisfacción en relación con los indicadores y factores de calidad de vida familiar

A continuación, se presentan los resultados descriptivos de la distribución de la muestra en relación a los 5 factores del Modelo de Calidad de Vida Familiar. La Tabla 2 muestra la frecuencia relativa expresada en el porcentaje de los factores de calidad de vida familiar en las áreas de necesidades, neutra y de fortalezas del MCVF, de acuerdo con la satisfacción que manifestaron los participantes.

La Tabla 2 muestra que el Rol Parental, la Interacción Familiar, y la Salud y Seguridad fueron los factores que tuvieron el mayor reporte de satisfacción por parte de las familias. Mientras que los factores de Recursos Generales y Apoyo a la Discapacidad tuvieron el mayor reporte de insatisfacción por parte de las familias. Los datos presentados en la tabla permiten evidenciar que globalmente las familias se perciben con una calidad de vida satisfactoria (categorías de satisfacción neutra y fuerte).

\section{Tabla 2}

Localización de los factores de calidad de vida familiar y las áreas del Mapa de Calidad de Vida Familiar $(n=40)$

\begin{tabular}{lccc}
\hline \multirow{2}{*}{\multicolumn{1}{c}{ Factor }} & \multicolumn{3}{c}{ Área MCVF (\%) } \\
\cline { 2 - 4 } & $\begin{array}{c}\text { Área } \\
\text { crítica }\end{array}$ & $\begin{array}{c}\text { Área } \\
\text { neutra }\end{array}$ & $\begin{array}{c}\text { Área } \\
\text { fuerte }\end{array}$ \\
\hline Interacción familiar & 13 & 25 & 62 \\
Rol parental & 9 & 23 & 68 \\
Salud y seguridad & 18 & 29 & 53 \\
Recursos generales & 38 & 28 & 34 \\
Apoyo para personas con & & & \\
discapacidad & 39 & 21 & 40 \\
\hline
\end{tabular}

Como se mencionó anteriormente, el Rol Parental fue uno de los factores que obtuvo los mejores resultados de satisfacción percibida, ubicándose el mayor reporte de satisfacción en el área fuerte del mapa con un (68\%), mientras que el (9\%) se ubicó en el área de las necesidades. De los diferentes ítems que componen este factor, se destaca la mayor satisfacción en los criterios relacionados con que "se enseñe a nuestro familiar con discapacidad cómo llevarse bien con otros" (ítem 13) (45\%), y en que "los miembros de la familia enseñemos a los niños como ayudar en el trabajo de la casa" (ítem 11) (43\%). Por el contrario, el ítem donde las familias percibieron mayor insatisfacción fue el (ítem 14), "que los adultos en mi familia tengamos la información necesaria para tomar decisiones acerca de nuestro familiar con discapacidad".

Así mismo, el factor de Interacción Familiar se ubicó dentro del mayor reporte de satisfacción por parte de las familias con un (62\%), contrariamente al $(13 \%)$ que fue valorado en el área crítica. Este factor está compuesto por 9 ítems, y fue el (ítem 7), el que se relaciona con que "los miembros de la familia muestren que se quieren y cuidan mutuamente", el que mostró la mayor satisfacción de las familias con un porcentaje de (80\%), seguido por el (ítem 1), "Mi familia disfruta del tiempo que pasa reunida", con un $(65 \%)$. Estos resultados demuestran que existe un buen clima familiar basado en las buenas relaciones entre los integrantes del grupo. Sin embargo, fue el (ítem 8), "que los miembros de mi familia tengamos buenas relaciones con la familia 
extensa", el que tuvo la calificación más baja de satisfacción (20\%).

El $53 \%$ de las familias reportaron el factor Salud y Seguridad en el área Fuerte del mapa. Mientras que el 29\% lo ubicó en el área neutra, y un $18 \%$ en el área crítica. Los ítems de este factor que obtuvieron el mayor reporte de satisfacción, fueron el (ítem 22), "Los miembros de la familia se cuidan unos a otros cuando alguno está enfermo" (83\%), y el (ítem 18), "Que mi familia sea físicamente sana" (73\%). Sin embargo, uno de los criterios que componen el factor de Salud y Seguridad, el (ítem 23), que se relaciona con que "la familia obtenga servicio de entidades de salud que conozcan sus necesidades individuales de salud", recibió una valoración de insatisfacción del 35\% que lo ubica en el área de necesidades

Por otro lado, la tabla muestra que el factor de Recursos Generales, que se relaciona con la obtención de ayuda externa y oportunidades para relacionarse con personas fuera de la familia, y el factor de Apoyo a Personas con Discapacidad, que se relaciona con las oportunidades con que cuenta el miembro de la familia con discapacidad para participar en diferentes actividades tales como educación, trabajo y tiempo libre, se ubicaron dentro del área crítica obteniendo los resultados más altos de insatisfacción (38\% y $39 \%$, respectivamente), en comparación con los otros factores de Calidad de Vida Familiar. Sin embargo, es necesario indicar que la inconformidad con estos factores se manifiesta en ítems específicos.

Para el caso del factor Recursos generales, los ítems que obtuvieron el mayor porcentaje de insatisfacción fueron aquellos relacionados con la situación económica: el (ítem 27), que indica que "Los miembros de mi familia cuenten con recursos para desplazarse hacia los sitios donde necesitan estar", y el (ítem 31), que se refiere a "Mi familia tenga la manera de controlar los gastos", con un porcentaje del $50 \%$ cada uno.

Mientras que en el factor Apoyo a Personas con discapacidad, los ítems reportados con la mayor insatisfacción (80\% cada uno), fueron los (ítem 38 y 39), que manifiestan que "Mi familia tenga el apoyo para conseguir los beneficios del gobierno que necesita el miembro de la familia con discapacidad" y que "Mi familia tenga apoyo de las entidades locales para conseguir los servicios que necesita el miembro de la familia con discapacidad", respectivamente.

\section{Discusión}

Los resultados de la presente investigación ofrecen información acerca de la satisfacción con la calidad de vida de familias que conviven con niños y adolescentes con discapacidad asociada a anomalías congénitas en el suroccidente colombiano.

El hallazgo más importante en este estudio fue que los factores de Calidad de Vida Familiar se ubicaron predominantemente en el área fuerte del MCVF, a excepción de las dimensiones de Recursos Generales y Apoyo a Personas con Discapacidad, que se ubicaron con reportes similares tanto en el área de necesidades, como en el área de fortalezas.

De acuerdo con lo anterior, es interesante detenerse a analizar dichos resultados tomando en consideración las tres variables que Zuna, Summers, Turnbull, Hu, y Xu, (2010) plantean como determinantes en la calidad de vida familiar; las variables individuales, de unidad familiar y las relacionadas con la actuación de servicios y programas.

En primer lugar, respecto a las variables individuales, no se hallaron estudios comparables en cuanto al grupo de edad y al origen de la discapacidad. Los más próximos para establecer comparaciones, corresponden a dos estudios desarrollados en Colombia que presentan hallazgos contrastantes. Córdoba et al. (2008), señalan que no existen diferencias significativas en el nivel de satisfacción de las familias en función de variables individuales como la edad y el tipo de discapacidad sobre calidad de vida familiar, en población con discapacidad menor de 18 años de edad. Ballesteros et al. (2006), identificaron que si bien en el análisis estadístico el diagnóstico de malformaciones congénitas no tuvo impacto en los dominios de calidad de vida de las cuidadoras de niños menores de dos años, tras un análisis cualitativo, se identificó que es necesario minimizar el impacto del primer 
momento en que se entrega información sobre el diagnóstico a la familia.

En este punto, es necesario considerar que las anomalías congénitas se presentan como determinantes de discapacidad (OMS, 2015). Esta situación anticipa que las familias enfrenten desde el momento del diagnóstico, una perspectiva de riesgo para morbilidad y mortalidad, substancialmente diferentes a las que enfrentan discapacidades con otros orígenes. En este sentido, es pertinente ahondar en la comprensión de estas dimensiones a través de investigaciones con un enfoque cualitativo.

Adicionalmente, los resultados de esta investigación demuestran que la madre de la persona con discapacidad es el familiar que asume el rol de cuidador principal, en concordancia con lo reportado por González del Yerro, CagigalGregorio, y Blas-Gómez (2013); Larrañaga et al. (2008); Zapata-Albán y Galarza-Iglesias, (2013); Córdoba et al. (2007). La madre, es quien además, se encarga de las labores del hogar, pese a que la mayoría de las familias están conformadas por más de tres personas. De esta manera, los reportes permiten concluir que existe una relación entre ser el cuidador principal y realizar las labores del hogar como ocupación prevalente, con una doble carga de trabajo (Burton-Amith, McVillyl, Yazbeck, Parmenter, \& Tsutsui, 2009) y una distribución inequitativa de las responsabilidades del hogar. Por lo tanto, se esperaría que esta sobrecarga por la doble condición: cuidador-ama de casa, tuviera repercusiones graves para la salud física y emocional de la madre y, en consecuencia, un desajuste en la dinámica del funcionamiento de la familia. Sin embargo, Ballesteros et al. (2006) y Córdoba et al. (2008) señalan que la variable género del cuidador no es una variable de influencia en el nivel de satisfacción con la calidad de vida familiar. Estos resultados podrían profundizarse con un estudio cualitativo, debido a que investigaciones sobre cuidado y mujer (Mier, Romeo, Canto, \& Mier, 2007), señalan que este rol ha sido asignado a través de la historia al género femenino, naturalizándolo y legitimándolo, hasta el punto de considerarse una obligación moral que las lleva a asumir la situación con conformismo, con un coste emocional tanto para ella, como para la familia.

En segundo lugar, se evidencia que los indicadores reconocidos como fortalezas de las familias, se relacionan con las variables de unidad familiar; mientras que aquellos que se identificaron como necesidades, se relacionan con las variables de la actuación de servicios y programas.

En ese sentido, las familias destacaron como principales fortalezas, ítems relacionados con las dinámicas internas de las familias; los ítems 11 y 13 del factor de Interacción familiar, el ítem 7 del factor Rol parental y el ítem 22 del factor Salud y seguridad. Estas fortalezas demuestran cohesión, corresponsabilidad en la toma de decisiones y buen manejo en la resolución de problemas en el entorno familiar; resultados que cobran importancia como recursos de la calidad de vida familiar de personas con discapacidad (Zuna, Turnbull, \& Summers, 2009), al reflejar la forma práctica con la que cuentan para responder a las necesidades de sus miembros, de articular su vida en común, y de ejercer una formación para la vida en el marco de valores como la solidaridad, el respeto y la sensibilidad por el otro.

Por otro lado, llama la atención el indicador con el que las familias manifestaron mayor necesidad, el ítem 14 del factor de Rol parental. En Colombia, particularmente en el ámbito de la salud, el acceso a información para tomar decisiones respecto a situaciones relacionadas con un familiar con anomalías congénitas, es limitado. Las anomalías congénitas son patologías complejas que requieren de profesionales especializados para su manejo, lo cual se dificulta en la ausencia de conocimientos clínicos sobre ellas (Bernal \& Suárez, 2008), y se refleja en la oportunidad de brindar servicios adecuados, entre ellos, asesoría genética a las familias de esta población, y en trazar políticas que se adecuen a sus necesidades. Esta situación ubica en un lugar de vulnerabilidad a las familias de personas con anomalías congénitas, al considerar que el acceso a información es apenas el primer nivel de participación ciudadana que permite a los sujetos integrarse para compartir los beneficios de la sociedad (Arntsein, 1969). 
Adicionalmente, los criterios de calidad de vida que se señalaron como insatisfechos, y que se incluyen en la variable de servicios y programas, también guardan relación con lo anteriormente mencionado acerca de la situación de las anomalías congénitas en Colombia. Las anomalías congénitas son patologías abordadas por profesionales especializados que ejercen labores en centros de referencia como la clínica Valle del Lili, ubicados en las principales ciudades del país, situación que implica para aquellos que viven en los alrededores de Cali, un desplazamiento que genera un gasto adicional a las familias en términos de transporte, y un desajuste en la dinámica diaria, relacionado con el cuidado del resto de la familia.

Así mismo, las políticas para el reconocimiento y atención de esta población, se encuentran en proceso de implementación, razón por la cual se ve enfrentada a barreras para acceder a diferentes servicios sociales; de ahí que uno de los criterios de mayor insatisfacción sobre la calidad de vida familiar en esta investigación, se relacione con el apoyo del gobierno para conseguir los beneficios que necesita la persona con discapacidad.

Finalmente, entre las limitaciones de este estudio, se encontró un tamaño reducido del grupo, que si bien corresponde a una muestra representativa de las personas con diagnósticos genéticos, atendidas en la consulta de genética clínica de una institución de referencia, la fuente de los casos se encuentra limitada a la población pediátrica, y no se obtiene representación de población en edad adulta. Incluso con una población menor de edad, es inevitable la influencia del sesgo de sobrevida en esta investigación. Los casos que conforman la muestra representan solo cohortes de nacimiento con una sobrevida alta, que puede resultar de una combinación de factores, por un lado los biológicos relacionados con la severidad y complejidad de las anomalías congénitas, y por otro lado, los relacionados con el acceso y uso de los servicios de salud de alta complejidad.

Otra limitación en el estudio, se relaciona con la inclusión de los criterios de calidad de vida familiar, que se ubicaron como medianamente satisfechos, debido a que en la versión original del MCVF, estos no se ubican en el área fuerte, ni en el área de necesidades, y se los deja por fuera de las consideraciones. En este sentido, es necesario desarrollar investigaciones que exploren la validez interna y el rendimiento del MCVF, cuando predominan las respuestas en categorías intermedias o neutras de satisfacción.

El mayor aprendizaje de este trabajo fue la confirmación de la necesidad del abordaje interdisciplinar e intersectorial, pero sobre todo, con la familia, para la construcción de una respuesta que logre satisfacer las necesidades de esta y de las personas con discapacidad en particular. Así mismo, un aprendizaje obtenido se relaciona con la experiencia que cada familia ha tenido a través de los años acerca de la discapacidad, al enfrentarse a obstáculos que no tienen su raíz en lo individual del sujeto, sino en las limitaciones que impone la sociedad para prestar servicios apropiados y para asegurar que sus necesidades sean tenidas en cuenta dentro de la organización social (Palacios, 2008), y que sirven como modelo para otras familias que apenas empiezan a enfrentarse a estas limitaciones.

Adicionalmente, se resalta el uso de la entrevista de Calidad de Vida Familiar en un proceso de investigación asociado al contexto clínico. En este sentido, permitió la recolección de información útil, tanto para el proyecto, como para la intervención, al generar el espacio de escucha y discusión entre los familiares sobre asuntos que difícilmente se manifiestan por la habituación de las actividades.

\section{Agradecimientos}

Los autores agradecemos a la Universidad Icesi, que financió, a través de recursos de la convocatoria interna, el proyecto titulado: "Estrategia de orientación a familias e instituciones educativas de niños y adolescentes en situación de discapacidad de origen genético a partir del modelo de calidad de vida familiar". A la Fundación Clínica Valle del Lili, y en especial a las familias de niños y jóvenes en situación de 
discapacidad, por su apoyo y participación en esta investigación.

\section{Referencias}

Arntsein, S. (1969). A Ladder of Citizen Participation. Journal of the American Planning Association, 35(4), 216-224.

Aya, V., \& Córdoba, L. (2013). Asumiendo juntos los retos: calidad de vida en familias de jóvenes con discapacidad intelectual. Revista Facultad de Medicina, 61(2), 155-166. Recuperado de http://www.scielo. org.co/pdf/rfmun/v61n2/v61n2a07.pdf

Ballesteros, B. P., Novoa Gómez, M. M., Muñoz, L., Suárez, F., \& Zarante, I. (2006). Calidad de vida en familias con niños menores de dos años afectados por malformaciones congénitas perspectiva del cuidador principal. Universitas Psychologica, 5(3), 457-474. Recuperado de http://www. scielo.org.co/pdf/rups/v5n3/v5n3a03.pdf

Bernal, J., \& Suárez, F. (2008). La carga de la enfermedad genética en Colombia, 1996-2025. Universitas Médica, 49(1), $12-28$.

Burton-Amith, R., McVilly, K., Yazbeck, M., Parmenter, T., \& Tsutsui, T. (2009). Quality of life of australian family carers: Implications for research, policy and practice. Journal of Policy $\mathcal{E}$ Practice in Intellectual Disabilities, 6(3), 189-198.

Córdoba, L., Gómez, J., \& Verdugo, M. A. (2008). Calidad de vida familiar en personas con discapacidad: un análisis comparativo. Universitas Psychologica, 7(2), 369-383. Recuperado de http://www.scielo. org.co/pdf/rups/v7n2/v7n2a06.pdf

Córdoba, L., Mora, A., Bedoya, A., \& Verdugo, M. (2007). Familias de adultos con discapacidad intelectual en Cali, Colombia, desde el modelo de calidad de vida. Psykhe, 16(2), 29-42. Recuperado de http://www.sc ielo.cl/pdf/psykhe/v16n2/art03.pdf

Córdoba, L., Verdugo, M. A., \& Gómez, J. (2006). Satisfacción con la calidad de vida en familias de niños y adolescentes con discapacidad en Cali, Colombia. En M. A. Verdugo, \& B. Jordán de Urríes (Coords.), Rompiendo inercias: Claves para avanzar (pp. 181-195). Salamanca: Amarú. Recuperado de http://sid.usal.es/idocs/F8/ART20386/c alidad_vida_familiar_249.pdf

EUROCAT. (2012). Special report: Congenital anomalies are a major group of mainly rare diseases. Recuperado de http://www.euroca t-network.eu/memberreg/

Fernández, A., Montero, D., Martínez, N., Orcasitas, J. R., \& Villaescusa, M. (2015). Calidad de vida familiar: marco de referencia, evaluación e intervención. Siglo creo, 46(2), 7-29. Recuperado de http://revistas.usal.es/index.php/0210-1 696/article/view/scero2015462729/15818

Fernández, N., \& Zarante, I. (2007). Prevalencia y escala pronóstico para malformaciones congénitas en Colombia: La responsabilidad de pediatras y neonatólogos. UCIN, 7(4), 28-32.

González del Yerro, A., Cagigal-Gregorio, V., Blas-Gómez, E. (2013). La calidad de vida de las familias de personas con discapacidad intelectual. Un estudio cualitativo realizado en la comunidad de Madrid. Revista Española de Orientación y Psicopedagogía, 24(1), 93-109.

Larrañaga, I., Martín, U., Bacigalupe, A., Begiristáin, J. M., Valderrama, M. J., \& Arregi, B. (2008). Impacto del cuidado informal en la salud y la calidad de vida de las personas cuidadoras: análisis de las desigualdades de género. Gac Sanit, 22(5), 443-450.

Mier, I., Romeo, Z., Canto, A., \& Mier, R. (2007). Interpretando el cuidado. Por qué cuidan solo las mujeres y qué podemos hacer para evitarlo. Revista de Servicios Sociales, 42, 29-38.

OMS. (2015). Anomalías congénitas. Recuperado de http://www.who.int/mediacentre/factsh eets/fs370/es/

Palacios, A. (2008). El modelo Social de la discapacidad: Orígenes, caracterización y plasmación en la convención internacional 
sobre los derechos de las personas con discapacidad. Madrid: Ediciones Cinca.

Park, J., Hoffman, L., Marquis, J., Turnbull, A., Poston, D., Mannan, H. ..., \& Nelson, L. L. (2003). Toward assessing family outcomes of service delivery: Validation of a family quality of life survey. Journal of Intellectual Disability Research, 47(4-5), 367-384.

Park, J., Turnbull, A., \& Turnbull, R. (2002). Impacts of poverty on quality of life in families with children with disabilities. Exceptional Children, 68(2), 151-170.

Poston, D., Turnbull, A., Park, J., Mannan, H., Marquis, J., \& Wang, M. (2003). Family quality of life: A qualitative inquiry. Mental Retardation, 41 (5), 313-328.

Resch, J., Mireles, G., Benz, M., Grenwelge, C., Peterson, P., \& Zhang, D. (2010). Giving Parents a Voice: A Qualitative Study of the Challenges Experienced by Parents of Children with Disabilities. Rehabilitation Psychology, 55(2), 139-150.

Schalock, R., \& Verdugo, M. (2002). The concept of quality of life in human services: A handbook for human service practitioners. Washington, DC: American Association on Mental Retardation.

Schalock, R., \& Verdugo, M. (2003). Calidad de vida. Manual para profesionales de la salud, educación y servicios sociales. (M. A. Verdugo, \& C. Jenaro, Trad). Madrid: Alianza. (Originalmente publicado en 2002).

Summers, J. A., Hoffman, L., Maquis, J., Turnbull, A., \& Poston, D. (2005). Relationship between parent satisfaction regarding partnerships with professionals and age of child. Topics in Early Childhood Special Education, 25(1), 48-58.

Vanegas, J., \& Gil, L. (2007). La discapacidad, Una mirada desde la teoría de sistemas y el modelo biopsicosocial. Hacia la promoción de la salud, 12, 51-61.

Verdugo, M. A., Córdoba, L., \& Gómez, J. (2006). Adaptación y validación al español de la Escala de Calidad de Vida Familiar (ECVF). Siglo Cero, 37(2), 41-46.
Zapata-Albán, M., \& Galarza-Iglesias, A. (2013). Caracterización de una población en situación de discapacidad intelectual desde los dominios de la salud y los dominios relacionados con la salud, con miras a un proceso de inclusión laboral. Revista de la Facultad de Medicina, 61 (2), 145-163.

Zuna, N., Summers, J. A., Turnbull, A. P., $\mathrm{Hu}, \mathrm{X} ., \quad \& \mathrm{Xu}$, S. (2010). Theorizing about family quality of life. In R. Kober (Ed.), Enhancing the quality of life of people with intellectual disabilities (pp. 241-278). Netherlands: Springers.

Zuna, N. I., Turnbull, A., \& Summers, J. A. (2009). Family quality of life: Moving from measurement to application. Journal of Policy and Practice in Intellectual Disabilities, 6(1), 25-31.

\section{Notas}

1 Salario mínimo legal vigente en Colombia para 2013: $\$ 589500(1 \mathrm{USD}=2541 \mathrm{COP})$

* Artículo de investigación. 